\title{
Improvement of Stopband Performance in Parallel-Coupled Bandpass Filters Using Quasi-Lumped Elements
}

\author{
Zhurbenko, Vitaliy; Krozer, Viktor; Meincke, Peter
}

Published in:

IEEE MTT-S International Microwave Symposium Digest

Link to article, DOI:

10.1109/MWSYM.2008.4633224

Publication date:

2008

Document Version

Publisher's PDF, also known as Version of record

Link back to DTU Orbit

Citation (APA):

Zhurbenko, V., Krozer, V., \& Meincke, P. (2008). Improvement of Stopband Performance in Parallel-Coupled Bandpass Filters Using Quasi-Lumped Elements. In IEEE MTT-S International Microwave Symposium Digest (pp. 547-550). IEEE. https://doi.org/10.1109/MWSYM.2008.4633224

\section{General rights}

Copyright and moral rights for the publications made accessible in the public portal are retained by the authors and/or other copyright owners and it is a condition of accessing publications that users recognise and abide by the legal requirements associated with these rights.

- Users may download and print one copy of any publication from the public portal for the purpose of private study or research.

- You may not further distribute the material or use it for any profit-making activity or commercial gain

- You may freely distribute the URL identifying the publication in the public portal 


\title{
Improvement of Stopband Performance in Parallel-Coupled Bandpass Filters Using Quasi-Lumped Elements
}

\author{
Vitaliy Zhurbenko, Viktor Krozer, and Peter Meincke \\ Technical University of Denmark, Department of Electrical Engineering, ElectroScience, \\ Ørsteds Plads, building 348, 2800 Kgs. Lyngby, Denmark
}

\begin{abstract}
This paper is aimed at improving the rejection level as well as overcoming the bandwidth limitations for classical coupled-line filters. A planar microwave coupled-line filter employing a quasi-lumped element resonator considerably improving the stopband characteristics is presented. The proposed bandpass filter has a compact footprint, and exhibits good stopband rejection with no repeated passband at twice the center frequency in comparison with the traditional coupled-line filter. By introducing the quasi-lumped element resonator, two transmission zeros at upper and lower stopbands are created, with adjustable locations of transmission zeros for desired performance. The device is fabricated in standard thick-film manufacturing technology. Based on parallel-coupled line theory, the impedance inverter model for this device is developed. The passband filter centered at $5.5 \mathrm{GHz}$ with a $3 \mathrm{~dB}$ relative bandwidth of $60 \%$ is implemented with an area of approximately $\lambda / 6 \times 2 / 4$. Measured and simulated results exhibit good agreement.
\end{abstract}

Index Terms - Coupled transmission lines, microstrip components, microstrip filters, microwave filters.

\section{INTRODUCTION}

The size reduction and performance improvement of microwave filters remains a great challenge today [1]. Among various types of microstrip filters, one of the most common implementations is based on the quarter-wavelength coupledline sections [2]. It is widely used due to relatively small dimensions and simple analysis techniques [3, 4]. However, the out-of-band rejection level of the traditional parallelcoupled bandpass filter is degraded by spurious response at twice the center frequency.

The present work focuses on a modified filter topology based on coupled-line sections including transmission zeros. In order to achieve sharp rejection and suppressed spurious response at twice the center frequency the quasi-lumped element resonator is implemented. This concept is used to design a microstrip bandpass filter with two transmission zeros, which improves the selectivity of the proposed filter. The presented design procedure overcomes the $50 \%$ bandwidth limit, discussed in [5], for a coupled-line implementation. We also demonstrate that relaxed dimensions can be used for the coupled-line sections in comparison with traditional design, where the coupled-line gap is generally a critical parameter.

\section{DESIGN TECHNIQUE}

Fig. 1 shows the circuit of the proposed bandpass filter with two-zeros based on coupled-line sections and quasi-lumped element resonator. This resonator consists of two inductors $L_{l}$, $L_{3}$, and two capacitors $C_{2}, C_{4}$. On the final layout these lumped elements in Fig. 1 are realized in the form of microstrip components, taking advantage of their well predictable performance in a wide frequency range and omission of via-holes for shunt capacitors.

Coupled line sections are characterized by the even- and odd-mode characteristic impedances $Z_{0 e}, Z_{0 o}$ and their respective lengths. The elements $L_{c}$ are introduced for adjustment of the central frequency, which increases the degree of freedom during the design procedure.

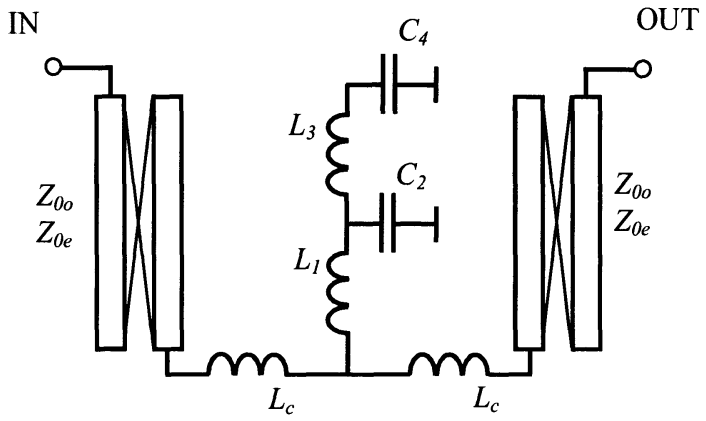

Fig. 1. Proposed filter circuit.

Inverter theory is applied with the purpose of developing an analytical model for the circuit in Fig. 1. It is common knowledge that the equivalent circuit of a coupled line section can be modeled as a series connection of transmission lines and $\mathrm{K}$-inverter [2]. Thus, by combining equivalent circuits of the coupled-line sections and quasi-lumped element resonator the equivalent circuit of the filter can be represented as shown in Fig. 2, where 


$$
Y=-j \frac{\omega\left(C_{4}-C_{2}\left(\omega^{2} L_{3} C_{4}-1\right)\right)}{\omega^{2} L_{1}\left(C_{4}-C_{2}\left(\omega^{2} L_{3} C_{4}-1\right)\right)+\omega^{2} L_{3} C_{4}-1},
$$

is the equivalent shunt conductance of the quasi-lumped element resonator; $j=\sqrt{-1}$.

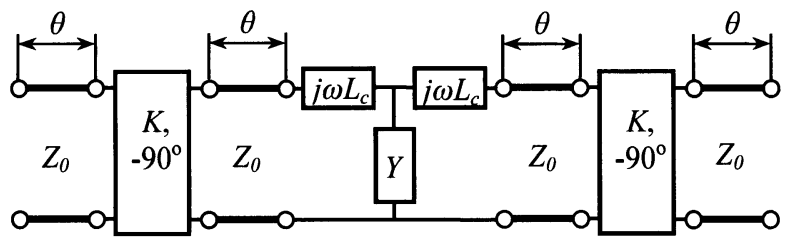

Fig. 2. K-inverter type equivalent circuit of the filter.

The characteristics of the filter can be derived from the ABCD matrix for the complete circuit, which consists of matrices for the filter components:

$$
\begin{aligned}
& {\left[\begin{array}{ll}
A & B \\
C & D
\end{array}\right]=} \\
& {\left[\begin{array}{ll}
A_{c l} & B_{c l} \\
C_{c l} & D_{c l}
\end{array}\right]\left[\begin{array}{cc}
1 & j \omega L_{c} \\
0 & 1
\end{array}\right]\left[\begin{array}{cc}
1 & 0 \\
Y & 1
\end{array}\right]\left[\begin{array}{cc}
1 & j \omega L_{c} \\
0 & 1
\end{array}\right]\left[\begin{array}{cc}
A_{c l} & B_{c l} \\
C_{c l} & D_{c l}
\end{array}\right],}
\end{aligned}
$$

where

$$
\begin{aligned}
& {\left[\begin{array}{ll}
A_{c l} & B_{c l} \\
C_{c l} & D_{c l}
\end{array}\right]=} \\
& {\left[\begin{array}{cc}
\cos \theta & j Z_{0} \sin \theta \\
\frac{j \sin \theta}{Z_{0}} & \cos \theta
\end{array}\right]\left[\begin{array}{cc}
0 & -j K \\
\frac{-j}{K} & 0
\end{array}\right]\left[\begin{array}{cc}
\cos \theta & j Z_{0} \sin \theta \\
\frac{j \sin \theta}{Z_{0}} & \cos \theta
\end{array}\right]}
\end{aligned}
$$

are the $A B C D$ parameters of the coupled line section with characteristic impedance $Z_{0}=\sqrt{Z_{0 e} \cdot Z_{0 o}}$ and electrical length $\theta=\theta_{e}=\theta_{o}$. Using this matrix representation, the electrical parameters of the filter can be derived, and analysis of the circuit can be performed. The voltage transfer function of the filter is determined as a product of the respective $\mathrm{ABCD}$ matrices of the individual elements.

The nature of the transmission zeros creation may be illustrated by considering the relation for the transmission coefficient of the quasi-lumped element resonator labeled with $\mathrm{Y}$ in Fig. 2:
$S_{21}=$

$$
\frac{p^{4} L_{1} L_{3} C_{2} C_{4}+p^{2}\left(L_{1}\left(C_{4}+C_{2}\right)+L_{3} C_{4}\right)+1}{\left(C_{4}+C_{2}\left(p^{2} L_{3} C_{4}+1\right)\right)\left(2 p^{2} L_{1}+50 p\right)+2 p^{2} L_{3} C_{4}+2} \text {, }
$$

where $p=j \omega$.

This relation is based on the model representation shown in Fig. 2 and relation (1). It can be easily seen that the resonator introduces two zeros in the transmission coefficient. The locations of the frequency transmission zeros can be found by solving the quadratic equation in the numerator (4) and taking into account frequency shifting due to $L_{c}$.

Therefore, the overall frequency response of the filter has two transmission zeros as shown in Fig. 3. Obviously, the locations of the transmission zeros for this filter may be adjusted by choosing suitable values for $L_{1}, L_{3}, C_{2}, C_{4}$.

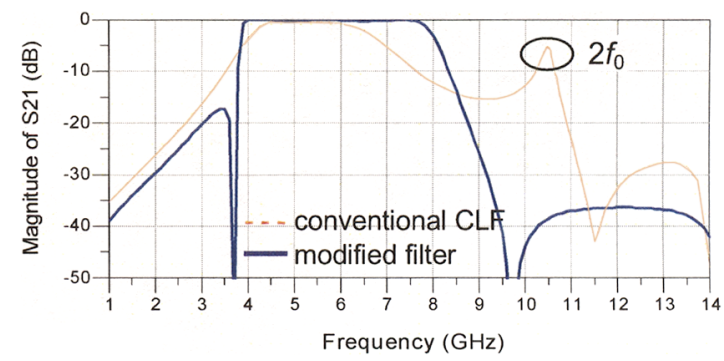

Fig. 3. Transmission characteristics for the traditional coupled-line filter (broken line) and bandpass filter based on quasi-lumped element resonator (solid line).

At the same time, due to selective properties of the quasilumped element resonator the spurious response at $2 f_{0}$ is suppressed. For reference, the response of the traditional coupled-line filter is shown in Fig. 3. It can be seen that the same coupled lines in a proposed configuration provide a wider bandwidth. It is known that the wider bandwidth requires a higher coupling level between lines in the traditional coupled-line filter. The coupling level is related to the gap dimension between lines. Therefore in practice, the fractional bandwidth of the traditional filter is limited by technological constraints. Considering the results presented in Fig. 3, one can conclude that the proposed structure requires lower coupling level for the same frequency bandwidth in comparison to the conventional one.

\section{FILTER IMPLEMENTATION AND RESULTS}

The proposed filter shown in Fig. 1 is designed for a frequency band $4-7 \mathrm{GHz}$ and fabricated on a substrate with the relative dielectric permittivity $\varepsilon_{r}=3.38$ and thickness $h=0.8 \mathrm{~mm}$. Values for the design parameters are presented in Table 1 . 
TABLE 1

PARAMETERS FOR THE FILTER SHOWN IN FIG. 1

\begin{tabular}{l|c|c|c|c|c|c|c}
\hline Parameter & $\begin{array}{c}Z_{0 e} \\
(\mathrm{Ohm})\end{array}$ & $\begin{array}{c}Z_{0 o} \\
(\mathrm{Ohm})\end{array}$ & $\begin{array}{c}L_{c} \\
(\mathrm{nH})\end{array}$ & $\begin{array}{c}L_{1} \\
(\mathrm{nH})\end{array}$ & $\begin{array}{c}L_{3} \\
(\mathrm{nH})\end{array}$ & $\begin{array}{c}C_{2} \\
(\mathrm{pF})\end{array}$ & $\begin{array}{c}C_{4} \\
(\mathrm{pF})\end{array}$ \\
\hline Value & 187 & 70 & 1.1 & 0.8 & 7.1 & 0.4 & 0.2 \\
\hline
\end{tabular}

Using values $Z_{0 e}, Z_{00}$ the coupled line dimensions can be determined from [2]. The layout of the filter is developed by transformation of the lumped components to equivalent microstrip elements (Fig. 4). The inductors in Fig. 1 are represented as microstrip lines with characteristic impedances $Z_{0(i)}$ and length $\ell_{(i)}$ using the relation

$$
L_{(i)}=\frac{2 \ell_{(i)} Z_{0(i)} \sqrt{\varepsilon_{r e f f}}}{c},
$$

where $c$ is the speed of light, $\varepsilon \quad f f$ is the effective dielectric constant, and $i$ is the index of the respective lumped component. The capacitors are transformed into microstrip patches using relation for a parallel plate capacitance with area $\mathrm{A}_{(i)}$ :

$$
C_{(i)}=\frac{\varepsilon_{0} \varepsilon_{r} A_{(i)}}{h}
$$

where $\varepsilon=8.85 \cdot 10^{-12} \mathrm{~F} / \mathrm{m}$.

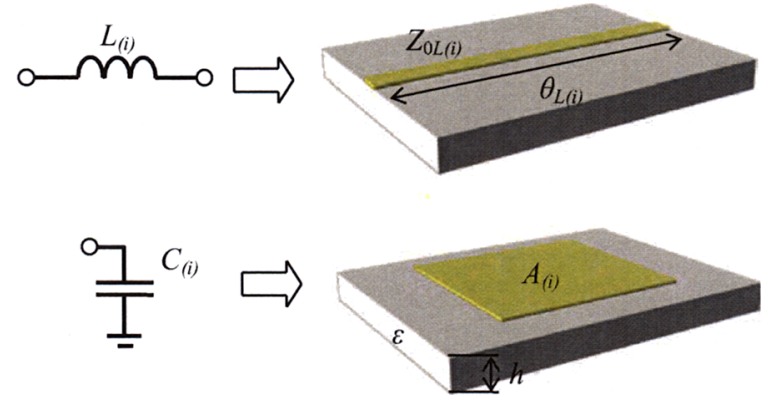

Fig. 4. The lumped components transformation.

Lumped elements, used for a model development, are frequency independent. To achieve better accuracy over a wide frequency band, the layout parameters are adjusted using well-developed microstrip transmission and coupled-line models.

Fig. 5 shows the geometry of the classical parallel coupledline filter and a modified bandpass structure which has an improved stopband characteristic and relaxed requirement to the coupling of the coupled lines.

Suitable filter layout is adopted to reduce the filter size resulting in a total occupied area of $\lambda / 6 \times \lambda / 4$. A picture of the filter is shown in Fig. 6. The coupled-line sections are bended to reduce the filter size, and the stub of length $l_{s}$, which is not represented in the schematic, is introduced to compensate for the length difference of the bended coupled line. The dimensions for the proposed structure, as illustrated in Fig. 6, are $l_{l}=5.5 \mathrm{~mm}, l^{\prime}{ }_{l}=2.6 \mathrm{~mm}, W_{l}=0.2 \mathrm{~mm}, S=0.2 \mathrm{~mm}$, $l_{s}=0.8 \mathrm{~mm}, l=0.8 \mathrm{~mm}$. The microstrip line denoted by $L_{l}$ has a length and a width of $0.6 \mathrm{~mm}$ and $0.2 \mathrm{~mm}$, respectively. The microstrip line denoted by $L$ has a length and a width of $5.3 \mathrm{~mm}$ and $0.2 \mathrm{~mm}$, respectively. The microstrip patches denoted by $C$ and $C$ have areas of $10.15 \mathrm{~mm}^{2}$ and $6.1 \mathrm{~mm}^{2}$, respectively. The method of moment simulations are used to take into account parasitic coupling between filter elements.

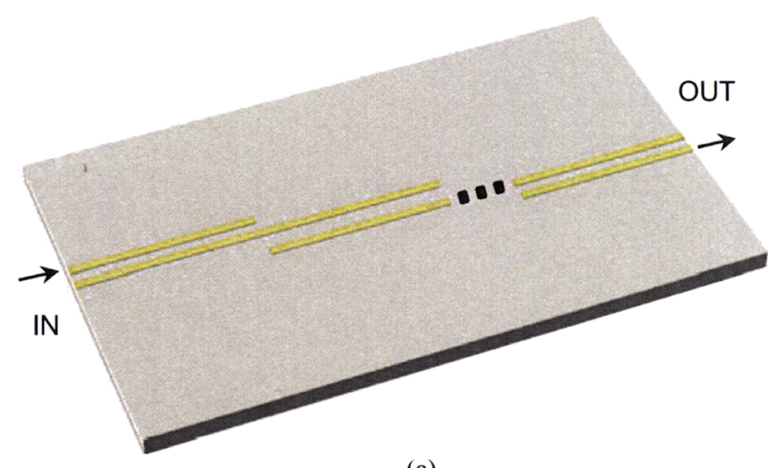

(a)

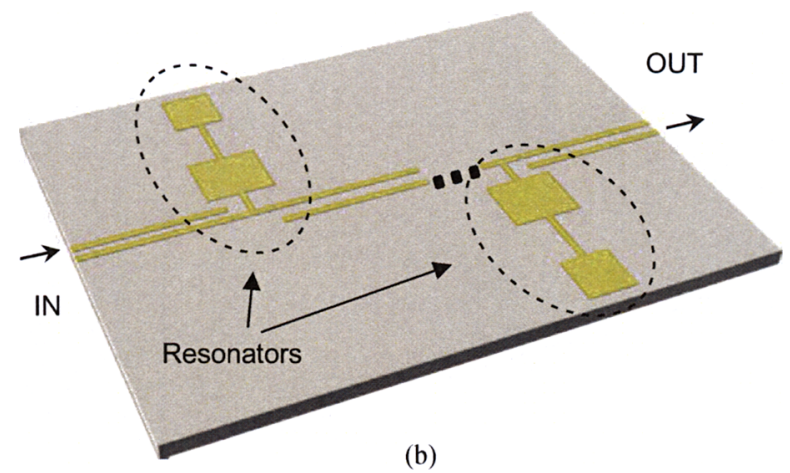

Fig.5. Parallel coupled-line bandpass filter: (a) conventional structure; (b) inclusion of resonant structures.

The simulation and measurement results are presented in Fig. 7.

Good agreement between measured and simulated results is observed over a wide frequency band. The minimum measured insertion loss is $0.8 \mathrm{~dB}$, including the connector and the feed line loss. Transmission zero at $3.7 \mathrm{GHz}$ is observed as expected. Transmission zero at upper stopband is slightly shifted to a higher frequency region. Though the filter is equivalent to a parallel-coupled bandpass filter, there is no 
repeated passband at $2 f_{0}$. The proposed structure exhibits a $3 \mathrm{~dB}$ fractional bandwidth of $60 \%$. The fractional bandwidth is defined as

$$
\delta f=\frac{\Delta f}{f_{0}}=2 \cdot \frac{\left(f_{\mathrm{h}}-f_{1}\right)}{\left(f_{\mathrm{h}}+f_{1}\right)},
$$

where $f_{\mathrm{l}}$ and $f_{\mathrm{h}}$ are the low and high end of the frequency band.

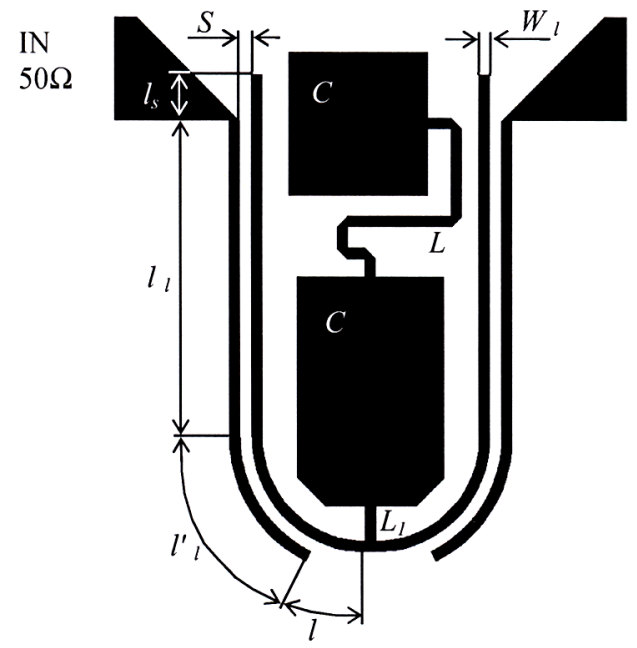

OUT

$50 \Omega$

(a)

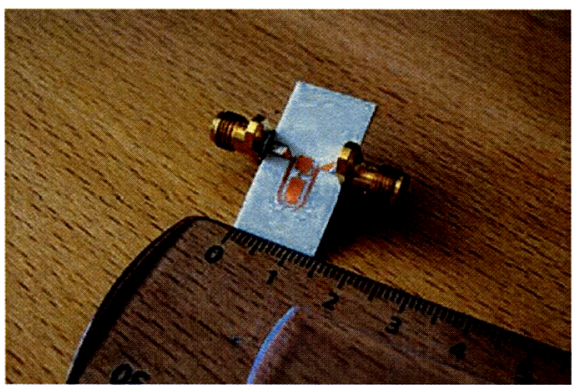

(b)

Fig. 6. Layout and photo of the filter based on coupled-line resonators and quasi-lumped element resonator.

The developed filter is intended to be used in a microwave camera for medical applications.

For some applications, higher stopband attenuation may be required. This can be achieved by increasing the order of the filter. The design of higher order filters is based on cascading several similar cells, as indicated schematically in Fig. 5.

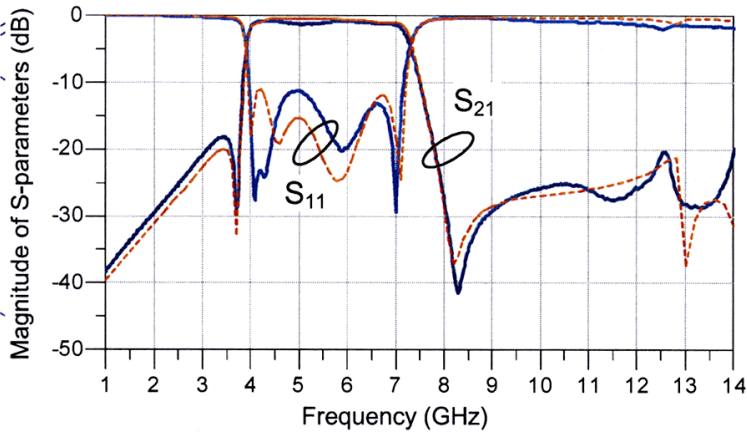

Fig. 7. Transmission and return loss characteristics for bandpass filter based on coupled-line resonators and quasi-lumped element resonator. The dotted line: calculated; the solid line: measured.

\section{Conclusion}

In this work, an effective method to realize two transmission zeros has been provided to achieve sharp rejection in a parallel-coupled bandpass filter. In addition, the proposed design exhibits no repeated passband at twice the center frequency. A filter has been designed and fabricated for the demonstration of the compactness and improved performance of this technique. The measured results are well matched with the simulated predictions. The fabrication technology is simple, inexpensive, and does not require via-holes. The filter exhibits a $3 \mathrm{~dB}$ bandwidth of $60 \%$ and an insertion loss of $0.8 \mathrm{~dB}$. The proposed method is suitable for a wideband as well as for narrowband applications.

\section{ACKNOWLEDGEMENT}

The authors would like to acknowledge the partial financial support by the Danish Ministry of Research and Education.

\section{REFERENCES}

[1] Jia-Sheng Hong, M. J. Lancaster, Microstrip Filters for RF/Microwave Applications. John Wiley \& Sons, Inc., 2001.

[2] R. Mongia, I. Bahl, P. Bhartia, RF and microwave coupled line circuits. Norwood: Artech House microwave library, 1999.

[3] V. Zhurbenko, V. Krozer, P. Meincke, "Miniature Microwave Bandpass Filter Based on EBG Structures", Proceedings of the 36th European Microwave Conference, pp 792-794, September 2006.

[4] G. Jaworski, V. Krozer, "A design of feeding network for a dual-linear polarization, stacked, probe-fed microstrip patch antenna array," Microwaves, Radar and Wireless Communications, vol. 2, pp. 473-476, May 2004.

[5] Jia-Sheng Hong, Hussein Shaman "An Optimum UltraWideband Microstrip Filter" Microwave And Optical Technology Letters, vol. 47, no. 3, pp. 230-233, November 5 2005. 